\title{
Multidimensional symptom clusters: An exploratory factor analysis in advanced chronic kidney disease.
}

\begin{abstract}
Aims: To explore the existence of symptom clusters in advanced chronic kidney disease.

Background: People with chronic kidney disease commonly report multiple symptoms. However, the complex relationships among these symptoms are still poorly understood.

Design: Cross-sectional

Methods: A total of 436 people with stage 4 and 5 chronic kidney disease were recruited from three tertiary hospitals during 2013-2014. Participants completed the Chronic Kidney Disease-Symptom Burden Index that assesses four symptom dimensions (occurrence, distress, severity and frequency) of 32 symptoms. Exploratory factor analysis was used to identify symptom clusters across each symptom dimension. Core symptoms in each cluster were determined based on stability across dimensions and clinical plausibility. A high cutoff of 0.50 for factor loading was used for all analyses.
\end{abstract}

Results: Five symptom clusters were consistently identified across all symptom dimensions: fluid volume symptoms, neuromuscular symptoms, gastrointestinal symptoms, sexual symptoms and psychological symptoms. Overall, clusters ranged from 2-10 symptoms. Several symptoms were also interconnected with multiple clusters. Fatigue cross-loaded on all five clusters, whereas sleep disturbance and restless leg symptoms cross-loaded across three clusters.

Conclusions: Adopting a symptom cluster approach has the potential to significantly advance symptom assessment and nursing care for people in advanced stages of chronic kidney disease. Routine clinical assessment and management strategies targeted at the cluster level should have synergistic effects in reducing symptoms. Fatigue is a pervasive symptom 
in advanced chronic kidney disease that is interconnected with global symptom burden, suggesting better management of symptom clusters may also reduce fatigue.

Keywords: Chronic kidney disease; nursing; management; symptom assessment; symptom clusters. 


\section{SUMMARY STATEMENT}

\section{Why is this research needed?}

- There is a high symptom burden associated with chronic kidney disease which is often under-recognised.

- Symptom cluster research in chronic kidney disease is beginning to emerge although most of the existing studies occur in the final stage of chronic kidney disease (i.e. end stage kidney disease).

- Information related to symptom clusters can assist in planning appropriate intervention strategies for patients with chronic kidney disease that lead to positive outcomes.

\section{What are the key findings?}

- This study builds on previous research by extending analysis to symptom clusters according to occurrence, severity, distress and frequency.

- Five symptom clusters were consistently identified across all symptom dimensions: fluid volume symptoms, neuromuscular symptoms, gastrointestinal symptoms, sexual symptoms and psychological symptoms.

- A novel finding was the presence of several interconnecting symptoms in chronic kidney disease (i.e. fatigue, sleep disturbance and restless leg), which may assist with explaining the high symptom burden in this population.

\section{How should the findings be used to influence policy/practice/research/ education?}

- Routine clinical assessment and management strategies targeted at the cluster level could have synergistic effects in reducing the burden of chronic kidney disease symptoms. 
- Targeted interventions directed to more than one symptom cluster may alleviate the overwhelming problems of fatigue experienced by people with chronic kidney disease. 


\section{INTRODUCTION}

The concept of a symptom cluster is newly introduced in nursing, particularly in renal nursing. Symptom clusters consist of 'Two or more symptoms that are related to each other that occur together. Symptom clusters are composed of stable groups of symptoms, are relatively independent of other clusters and may reveal specific underlying dimensions of symptoms. Symptoms in a cluster may or may not share the same aetiology' (Kim et al., 2005, p. 278). Symptoms are subjective experiences that affect everyday life (i.e. impact on activities of daily living) and nurses are often primarily responsible for the ongoing assessment and management of symptoms. Research suggests that symptom clusters independently predict functional status (Herr et al., 2015), quality of life (Amro, Waldum, Dammen, Miaskowski, \& Os, 2014; Thong et al., 2009) and mortality rate (Amro et al., 2015). Information about these correlations may be valuable to facilitate development of interventions that lead to improvements in patients' prognosis, functional status, quality of life and survival. Moreover, it can assist nurses in prioritising management actions by targeting the clusters that strongly predict patients' outcomes.

\section{Background}

Symptoms in chronic kidney disease (CKD) are still poorly understood, as most studies focuses on examining single symptoms such as fatigue, pain or depression (Almutary, Bonner, \& Douglas, 2013; Murtagh, Addington-Hall, \& Higginson, 2007). Previous studies indicate that the average number of CKD symptoms ranges between 6 to 20 different symptoms (Almutary et al., 2013) that is similar to those experienced in the terminal stages of cancer (Saini et al., 2006). Several factors could lead to occurrence of these symptoms such as the disease itself, treatment modalities or due to comorbid conditions. The existence of multiple symptoms could yield a concurrent group of symptoms that form a cluster (Dodd, Miaskowski, \& Lee, 2004). 
Symptom cluster research in CKD is beginning to emerge (Amro et al., 2014; Amro et al., 2015; Jablonski, 2007; Lee \& Jeon, 2015; Thong et al., 2009; Yu, Huang, \& Tsai, 2012), although most of these studies occur in the final stage of CKD (i.e. end stage kidney disease). Jablonski (2007) assessed 11 physical symptoms among 130 people receiving haemodialysis and identified four symptom clusters (energy/vitality, cardiac related problems, pain/comfort and problems related to the gastrointestinal system). Yu et al. (2012) also reported four symptom clusters (energy and sensory discomfort, gastrointestinal and cardiac-pulmonary symptom cluster, cardiovascular cluster and electrolyte imbalance cluster). Two further studies have found symptom clusters among people receiving dialysis therapy (mixed group of both haemodialysis and peritoneal dialysis) using the same instrument (i.e. symptoms from the Kidney Disease Quality of Life - Short Form [KDQOL-SF]) (Amro et al., 2014; Thong et al., 2009). Both studies found three similar symptom clusters (i.e. uraemic symptoms, neuromuscular problems and skin problems) and a strong association between these clusters and the quality of life. Interestingly, a uraemic symptom cluster was also reported as an independent predictor of mortality among those receiving dialysis (Amro et al., 2015). One recent study assessed 17 symptoms using the Patient Outcome Scale in 143 people with CKD stages 2-4, finding 5 symptom clusters (energy insufficiency and pain, skin problems, emotional instability and uraemia, circulatory problems and anaemia, neurological and bowel problems) (Lee \& Jeon, 2015).

Drawing clear conclusions about CKD symptom clusters from previous studies is difficult for several reasons. Research to date is mostly limited to the dialysis population (Amro et al., 2014; Jablonski, 2007; Thong et al., 2009; Yu et al., 2012), from small sample sizes (Jablonski, 2007; Lee \& Jeon, 2015; Yu et al., 2012) and measuring mostly physical symptoms (ranging from 11-17 symptoms) (Amro et al., 2014; Jablonski, 2007; Lee \& Jeon, 2015; Thong et al., 2009; Yu et al., 2012). Studies using the symptom list from the KDQOL- 
SF also omit fatigue and psychological symptoms, both highly prevalent symptoms (Amro et al., 2014; Thong et al., 2009). In addition, existing studies examine symptoms from one dimension (typically distress) rather than from multiple dimensions (i.e. occurrence, severity, frequency and distress) (Amro et al., 2014; Jablonski, 2007; Lee \& Jeon, 2015; Thong et al., 2009; Yu et al., 2012), which ignores the complexity of the symptom experience (Jablonski, 2007).

\section{Conceptual framework}

This study was informed by the Theory of Unpleasant Symptoms (TUS) that comprises three concepts: influencing factors, symptom experience and consequence (Lenz, Pugh, Milligan, Gift, \& Suppe, 1997). Symptom experience conceptually addressing the multidimensional feature of symptoms because a symptom can be characterised by not only its occurrence (i.e. prevalence), but also by its level of distress, severity and frequency (Lenz et al., 1997). As people with CKD experience a range of symptoms, examining the possible correlations among symptoms from multidimensional perspectives can improve the insight into clustering of symptom and the potential underlying aetiology.

\section{THE STUDY}

Aim

This study sought to explore symptom clusters based on different symptom dimensions (occurrence, distress, severity and frequency) in the most burdensome stages of CKD.

Design

A cross-sectional design was used in this study.

\section{Participants}


A convenience sample of people with advanced CKD was recruited from major kidney centres and nephrology clinics of three tertiary hospitals in the western region of Saudi Arabia from July 2013 - February 2014. Participants were adults diagnosed with stage 4 and $5 \mathrm{CKD} \quad\left(\mathrm{eGFR}<30 \mathrm{mls} / \mathrm{min} / 1.73 \mathrm{~m}^{2}\right)$, or currently receiving dialysis, either haemodialysis or peritoneal dialysis. The estimated Glomerular Filtration Rate (eGFR) for non-dialysis patients (stage 4 and 5) was calculated based on the most recent creatinine level using the CKD-EPI creatinine equation (Johnson et al., 2012). Generally, for factor analysis, a sample size of 300 is considered acceptable (Tabachnick \& Fidell, 2014). However, we sought to recruit at least 320 patients based on a 10:1 ratio (Costello \& Osborne, 2005).

\section{Data collection}

Eligible participants were initially identified by nephrologists and dialysis nurse managers. Self-report data were collected through in-person distribution and other data were extracted from dialysis charts and hospital files.

\section{Measures}

\section{Demographic and disease characteristics}

Demographic characteristics that could potentially influence the individual's symptom experiences were assessed. The number of co-morbidities was also estimated using Davies et al.'s co-morbidity index (Davies et al., 2002). Details of the procedure and participants' demographic and clinical characteristics have been reported previously (Almutary, Bonner, \& Douglas, 2016).

\section{CKD Symptoms}

Symptom clusters in CKD patients was assessed using the CKD Symptom Burden Index (CKD-SBI) (Almutary, Bonner, \& Douglas, 2015). The CKD-SBI was adapted from the Dialysis Symptom Index (Weisbord et al., 2004) to assess the occurrence, distress, 
severity and frequency of $32 \mathrm{CKD}$ symptoms during the past 4 weeks. The occurrence dimension assessed the presence or absence of symptoms (yes/no) and ranged between 0-32 symptoms. Other symptom dimensions (distress, severity and frequency) are rated on a 0-10 numerical rating scale. Participants rate the 'distress' subscale from none to very much, 'severity' from none to very severe and 'frequency' from never to constant.

\section{Ethical considerations}

This study was approved by the Queensland University of Technology Human Research Ethics Committees, King Abdulaziz University Hospital and Jeddah Research Centre ethics committees. The study was explained verbally and in writing prior to obtaining voluntary written consent.

\section{Statistical Analysis}

Analyses were conducted using IBM SPSS version 21 (Chicago, IL, USA) and Mplus version 7.11 (Muthen \& Muthen, Los Angeles, CA). A score of zero was assigned for symptom distress, severity and frequency of symptoms if the symptom was not present, to retain all cases in the factor analyses. First, a correlation matrix between symptoms for each symptom dimension was generated and examined to ensure sufficient correlations among the symptoms for factor analysis (Hair, black, Babin, \& Anderson, 2014). Also, the factorability of the data were evaluated against a Kaiser-Meyer-Olkin (KMO) value > 0.60 and the significance of Bartlett's test of Sphericity (Hair et al., 2014).

The same analysis was conducted for each symptom dimension (occurrence, distress, severity and frequency). Exploratory factor analyses (EFAs) were conducted to identify symptom clusters from $32 \mathrm{CKD}$ symptoms. Oblique rotation was conducted to simplify extraction and allow for correlations between extracted factors because symptoms are likely to be correlated with each other (Hair et al., 2014; Skerman, Yates, \& Battistutta, 2009). Weighted least squares estimation with a mean and variance adjustment was used for factor 
extraction based on occurrence ratings as the recommended extraction method for binary data, while principal axis factoring was used for the other symptom dimensions because this method does not require distributional assumption and has been commonly used in symptom cluster research (Skerman et al., 2009).

The number of factors (clusters) to be extracted was informed by examining factors with eigenvalues greater than 1 , examining the scree plot and investigating additional factors (Skerman et al., 2009; Tabachnick \& Fidell, 2014). For the binary data (occurrence), 3 types of fit indices were also assessed to determine the appropriate number of factors, including absolute fit (the $p$ value for a chi-square statistic should be $>0.05$ ), fit adjusting for model parsimony (root mean square error of approximation (RMSEA) should be $<0.06$ and standardized root mean square residual $(\mathrm{SRMR})<0.08)$ and comparative fit measures Comparative Fit Index (CFI) and the Tucker Lewis Index (TLI) should be > 0.90) (Hair et al., 2014). The decision about the final number of the factors was taken after we achieved the most parsimonious and clinically meaningful symptom clusters. The communalities of the symptoms were assessed and the solutions were compared after excluding those symptoms with low communalities from analyses, because inclusion of low inter-item correlations in the factor analysis could affect the correlation coefficient and magnitude of the loadings (Costello \& Osborne, 2005).

Both pattern and structure coefficients were assessed and interpreted for each analysis until a parsimonious structure was ensured. Interpretation of structure coefficients considers the complex relationships among the symptoms, which may indicate different sources for the underlying construct of how symptoms group together (Skerman, Yates, \& Battistutta, 2012). A minimum cutoff of 0.50 for factor loading was used for all analyses to improve interpretation of clusters and this has been considered essential for practical significance (Hair et al., 2014). As symptoms are complex and likely to be cross-loaded on more than one 
cluster, the decision to retain the symptom in one cluster was based on the significance of loading on one factor (with at least 0.20 difference between factors) and the conceptual and clinical relevance of symptoms. However, when a symptom was cross-loaded similarly and had large factor loadings across multiple clusters, it was retained in each relevant cluster. We have named these symptoms that are associated with multiple clusters as interconnecting symptoms.' Finally, the symptoms in each cluster were compared across each symptom dimension and stable symptoms (core symptoms) in each cluster were determined. The final clusters were defined after reviewing their face validity and clinical plausibility, based on the literature and authors' clinical experience. Each cluster was assigned a name to reflect the possible underlying aetiology. Supplementary file 1 summarizes the statistical methods used to identify symptom cluster.

\section{Validity and reliability/ Rigour}

This study used a validated instrument to explore symptom clusters among people with CKD. Good psychometric properties of the CKD-SBI were previously demonstrated with internal consistency ( $\alpha=0.91)$, discriminative and convergent validity of CKD-SBI (Almutary et al., 2015). In addition, a consistent technique was used during the study. The instruments were administered and collected by one of the researchers (first author). Assistance was provided by the same researcher (if required) who read out each item and possible responses. This technique assisted with maintaining a consistent approach during data collection and ensured that participants received the same instructions.

\section{RESULTS}

\section{Demographic and symptom characteristics}

Table 1 presents a summary of the demographic characteristics of the sample. Of the 436 people with $\mathrm{CKD}$, the mean age was 48 years (SD 14.86), $53 \%$ were men, $75.5 \%$ were 
receiving dialysis and $24.5 \%$ were non-dialysis patients. The majority of the participants were receiving haemodialysis treatment (65.8\%). Diabetes (31.4\%) and hypertension $(33.7 \%)$ were the main causes of CKD. Half of the participants suffered from at least two comorbid conditions.

The descriptive results of symptom burden have been reported in a previous paper (Almutary et al., 2016). Briefly, participants reported an average of 13.01 (SD 7.67) CKDrelated symptoms. With a maximum total score of 320 (possible range 0-320), the mean score for symptom distress was 57.55 (SD 52.27), symptom severity was 55.45 (SD 51.74) and symptom frequency was 58.76 (SD 50.48). The five most common symptoms were fatigue (77.3\%), bone or joint pain $(60.3 \%)$, pruritus $(59.6 \%)$, decreased appetite $(50.5 \%)$ and trouble falling asleep (49.1\%). 
Table 1 Sample Characteristics $(N=436)$

\begin{tabular}{ll}
\hline Variable & $n(\%)$ \\
\hline Age & \\
$M$ & $48.29(14.86)$ \\
Range & $18-87$ \\
$18-44$ & $168(38.5)$ \\
$45-64$ & $181(41.5)$ \\
$\geq 65$ & $66(15.1)$ \\
Gender & $231(53)$ \\
Male & $205(47)$ \\
Female & \\
Marital status & $176(40.4)$ \\
Not Married & $260(59.6)$ \\
Married & \\
Educational status & $235(53.9)$ \\
Less than secondary & $105(24.1)$ \\
Secondary & $94(21.6)$ \\
College or above & \\
Employment status & $222(50.9)$ \\
Employed & $130(29.8)$ \\
Unemployed & $84(19.3)$ \\
Retired & \\
CKD Stages \& treatment modalities & $69(15.8)$ \\
Stage 4 (non-dialysis) & $38(8.7)$ \\
Stage 5 (non-dialysis) & $42(9.6)$ \\
PD & $287(65.8)$ \\
HD & $182(41.7)$ \\
Comorbid conditions & $224(51.4)$ \\
0 & $30(6.9)$ \\
1-2 & \\
Z 3 & $137(31.4)$ \\
Causes of CKD & $147(33.7)$ \\
Diabetic nephropathy & $30(6.9)$ \\
Hypertensive nephropathy & $59(13.5)$ \\
Primary glomerular disease & \\
Unknown aetiology & \\
Others & \\
\hline & \\
&
\end{tabular}

Percentages vary depending on missing data. HD: haemodialysis, PD: peritoneal dialysis 


\section{Symptom clusters}

Data factorability was demonstrated by a KMO statistic of $\geq 0.90$ and a significant Bartlett's test of sphericity $(p<0.001)$ for each symptom dimension. Based on the initial solutions, 8 factors had eigenvalues greater than 1, in each symptom dimension. However, examination of the scree plot suggested that a 5-factor solution was likely to be the most appropriate solution for each symptom dimension (i.e. distress, severity and frequency). In addition, analysis of 4-, 5-, 6- and 7-factor extractions revealed that a 5-factor extraction was the most parsimonious structure and the most appropriate based on the factor interpretation. For symptom occurrence, on the basis of the fit indices of each model, a 5-factor solution also revealed the best fit $(\mathrm{RMSEA}=0.05[90 \%$ confidence interval $=0.04-0.05], \mathrm{SRMR}=0.06$, $\mathrm{CFI}=0.96, \mathrm{TLI}=0.95)$. There were no differences in the results found when excluding the symptoms with low communalities (e.g. nocturia and constipation), so all symptoms were retained in all analyses.

Five symptom clusters were identified based on occurrence, distress, severity and frequency of symptom after interpretation of both pattern and structural coefficients (see Table 2). Examining the pattern coefficients revealed symptoms were loaded only on one distinctive cluster, whereas examining the structure coefficients revealed more symptoms with high loading values and that were conceptually relevant to each cluster (see Table 3). Overall, clusters ranged from 2-10 symptoms across all symptom dimensions. These symptoms in each cluster reflected the core symptoms, plausible underlying constructs and have been labeled accordingly. These clusters were named 'fluid volume symptoms', 'gastrointestinal symptoms', 'neuromuscular symptoms', 'sexual symptoms' and 'psychological symptoms.' Analysis shows that two clusters (fluid volume symptoms and psychological symptoms) had a negative loading based on symptom severity ratings (i.e. 
symptoms with the lowest severity ratings on scale $0-10$ are grouped together). The correlations between the FIVE symptom clusters ranged from 0.16-0.59.

Table 2 Chronic Kidney Disease Symptom Clusters

\begin{tabular}{|c|c|}
\hline Cluster & Core symptoms ${ }^{\mathrm{a}}$ \\
\hline \multirow[t]{5}{*}{ Fluid volume symptoms } & Cough \\
\hline & Shortness of breath \\
\hline & Chest pain \\
\hline & Light headedness or dizziness \\
\hline & Difficulty concentrating \\
\hline \multirow[t]{2}{*}{ Neuromuscular symptoms } & Muscle soreness \\
\hline & Numbness or tingling in feet \\
\hline \multirow[t]{2}{*}{ Sexual symptoms } & Decreased interest in sex \\
\hline & Difficulty becoming sexually aroused \\
\hline \multirow[t]{5}{*}{ Psychological symptoms } & Feeling anxious \\
\hline & Worrying \\
\hline & Feeling sad \\
\hline & Depression \\
\hline & Feeling nervous \\
\hline \multirow[t]{2}{*}{ Gastrointestinal symptoms } & Vomiting \\
\hline & Nausea \\
\hline
\end{tabular}

${ }^{a}$ Core symptoms are stable across all symptom dimensions using pattern or structural factor loading cut-off $>0.50$ 


\section{Fluid volume symptom cluster}

The core symptoms in the fluid volume symptom cluster were cough, shortness of breath, chest pain, light headedness or dizziness and difficulty concentrating. These five symptoms were stable and existed across all symptom dimensions and had a large structural coefficient $>0.50$ (see Table 2). However, this cluster also comprised several groupings of symptoms ranging from 6 to 10 , according to the dimension of the symptom (Table 3 ).

\section{Gastrointestinal symptom cluster}

The findings showed that the gastrointestinal symptom cluster comprised the pair of nausea and vomiting as core symptoms across all dimensions. Decreased appetite and diarrhoea were also related to this cluster according to different symptom dimensions (Table 3).

\section{Neuromuscular symptom cluster}

The neuromuscular symptom cluster ranged from 3-7 symptoms (Table 3), although muscle soreness and numbness or tingling in feet were stable when the cluster was determined using different symptom dimensions. Muscle cramps had high significant loading in this cluster except when using distress ratings (loadings ranged from $0.89-0.58$ ). Also bone or joint pain loaded onto this cluster according to both severity and frequency of symptoms.

\section{Sexual symptom cluster}

The sexual symptom cluster consisted of two symptoms that reflected decreased interest in sex and difficulty becoming sexually aroused. These symptoms were stable across all dimensions of symptoms. Both of these symptoms loaded highly onto this cluster $(\geq 0.78)$. 


\section{Psychological symptom cluster}

A group of five psychological symptoms emerged as a cluster. All of these symptoms had high factor loadings $(\geq 0.62)$; however, feeling anxious and worrying had the highest loading. One additional symptom (irritability) was associated with this cluster when determined according to symptom occurrence. 
Table 3 CKD Symptom Clusters Based on Occurrence, Distress, Severity and Frequency of 32 Symptoms

\begin{tabular}{|c|c|c|c|c|c|c|c|}
\hline \multicolumn{2}{|l|}{ Occurrence } & \multicolumn{2}{|l|}{ Distress } & \multicolumn{2}{|l|}{ Severity } & \multicolumn{2}{|l|}{ Frequency } \\
\hline Symptom & Loading $^{\text {a }}$ & Symptom & Loading $^{\text {a }}$ & Symptom & Loading $^{\text {a }}$ & Symptom & Loading a $^{\text {a }}$ \\
\hline \multicolumn{8}{|c|}{ Fluid volume symptoms cluster } \\
\hline Dry skin & 0.77 & Dry skin & $\mathbf{0 . 8 0}$ & & & & \\
\hline Itching & 0.63 & Itching & 0.67 & & & Itching & 0.56 \\
\hline Cough & 0.77 & Cough & 0.62 & Cough & $0.58^{\mathrm{b}}$ & Cough & 0.62 \\
\hline Shortness of breath & 0.82 & Shortness of breath & 0.51 & Shortness of breath & $0.69^{b}$ & Shortness of breath & 0.68 \\
\hline Chest pain & 0.79 & Chest pain & 0.57 & Chest pain & $0.67^{b}$ & Chest pain & 0.61 \\
\hline Light headedness or dizziness & 0.75 & Light headedness or dizziness & 0.54 & Light headedness or dizziness & $0.68^{b}$ & Light headedness or dizziness & 0.69 \\
\hline Difficulty concentrating & 0.65 & Difficulty concentrating & 0.57 & Difficulty concentrating & $0.58^{\mathrm{b}}$ & Difficulty concentrating & 0.57 \\
\hline Dry mouth & 0.64 & Dry mouth & 0.60 & Decreased appetite & $0.53^{b}$ & Trouble staying asleep & 0.53 \\
\hline Swelling in legs & 0.68 & Trouble falling asleep & 0.51 & & & Restless leg syndrome & 0.60 \\
\hline Bone or joint pain & 0.61 & Headache & 0.54 & & & & \\
\hline \multicolumn{8}{|c|}{ Gastrointestinal symptoms cluster } \\
\hline Vomiting & $\mathbf{0 . 8 8}$ & Vomiting & 0.65 & Vomiting & 0.75 & Vomiting & 0.71 \\
\hline Nausea & 0.93 & Nausea & 0.73 & Nausea & 0.56 & Nausea & 0.67 \\
\hline & & Diarrhoea & 0.59 & Diarrhoea & 0.51 & & \\
\hline Decreased appetite & 0.64 & Decreased appetite & 0.52 & & & & \\
\hline \multicolumn{8}{|c|}{ Neuromuscular symptoms cluster } \\
\hline Muscle soreness & 0.92 & Muscle soreness & 0.60 & Muscle soreness & 0.77 & Muscle soreness & 0.76 \\
\hline Muscle cramps & 0.89 & Numbness or tingling in feet & $\mathbf{0 . 8 0}$ & Muscle cramps & 0.58 & Muscle cramps & 0.60 \\
\hline Numbness or tingling in feet & 0.67 & Restless leg syndrome & 0.75 & Numbness or tingling in feet & 0.65 & Numbness or tingling in feet & 0.59 \\
\hline & & & & Restless leg syndrome & 0.52 & Bone or joint pain & 0.62 \\
\hline & & & & Bone or joint pain & 0.74 & & \\
\hline & & & & Trouble falling asleep & 0.54 & & \\
\hline & & & & Feeling tired or lack of energy & 0.54 & & \\
\hline \multicolumn{8}{|c|}{ Sexual symptoms cluster } \\
\hline Decreased interest in sex & 0.99 & Decreased interest in sex & 0.98 & Decreased interest in sex & 0.93 & Decreased interest in sex & 0.92 \\
\hline $\begin{array}{l}\text { Difficulty becoming sexually } \\
\text { aroused }\end{array}$ & 0.95 & $\begin{array}{l}\text { Difficulty becoming sexually } \\
\text { aroused }\end{array}$ & 0.78 & $\begin{array}{l}\text { Difficulty becoming sexually } \\
\text { aroused }\end{array}$ & 0.90 & $\begin{array}{l}\text { Difficulty becoming sexually } \\
\text { aroused }\end{array}$ & $\mathbf{0 . 8 9}$ \\
\hline \multicolumn{8}{|c|}{ Psychological symptoms cluster } \\
\hline Feeling anxious & 0.90 & Feeling anxious & $\mathbf{0 . 7 9}$ & Feeling anxious & $0.84^{b}$ & Feeling anxious & 0.74 \\
\hline Worrying & 0.89 & Worrying & 0.72 & Worrying & $0.74^{b}$ & Worrying & 0.73 \\
\hline Feeling sad & 0.81 & Feeling sad & 0.71 & Feeling sad & $0.74^{b}$ & Feeling sad & 0.64 \\
\hline Depression & 0.82 & Depression & 0.70 & Depression & $0.72^{b}$ & Depression & 0.62 \\
\hline Feeling nervous & 0.88 & Feeling nervous & 0.64 & Feeling nervous & $0.69^{b}$ & Feeling nervous & 0.72 \\
\hline Irritability & 0.66 & & & & & & \\
\hline
\end{tabular}

${ }^{a}$ Bolded symptoms indicate the symptom in each cluster that exist on the pattern matrix with loading > 0.50; Non-bolded symptoms indicate symptoms with structure coefficients $>0.5 ;{ }^{\mathrm{b}}$ Symptoms with a negative loading. 


\section{Inter-connecting symptoms}

Examining the structural coefficients showed that several symptoms cross-loaded similarly in multiple clusters (Figure 1), which we have described as inter-connecting symptoms. Fatigue cross-loaded in all five symptom clusters with high loading values that ranged from $0.51-0.61$. Symptoms related to sleep disturbance (trouble falling asleep and trouble staying asleep) and restless leg cross-loaded in three symptom clusters (i.e. fluid volume symptoms, neuromuscular symptoms and psychological symptoms) (loadings ranged from $0.50-0.62)$.

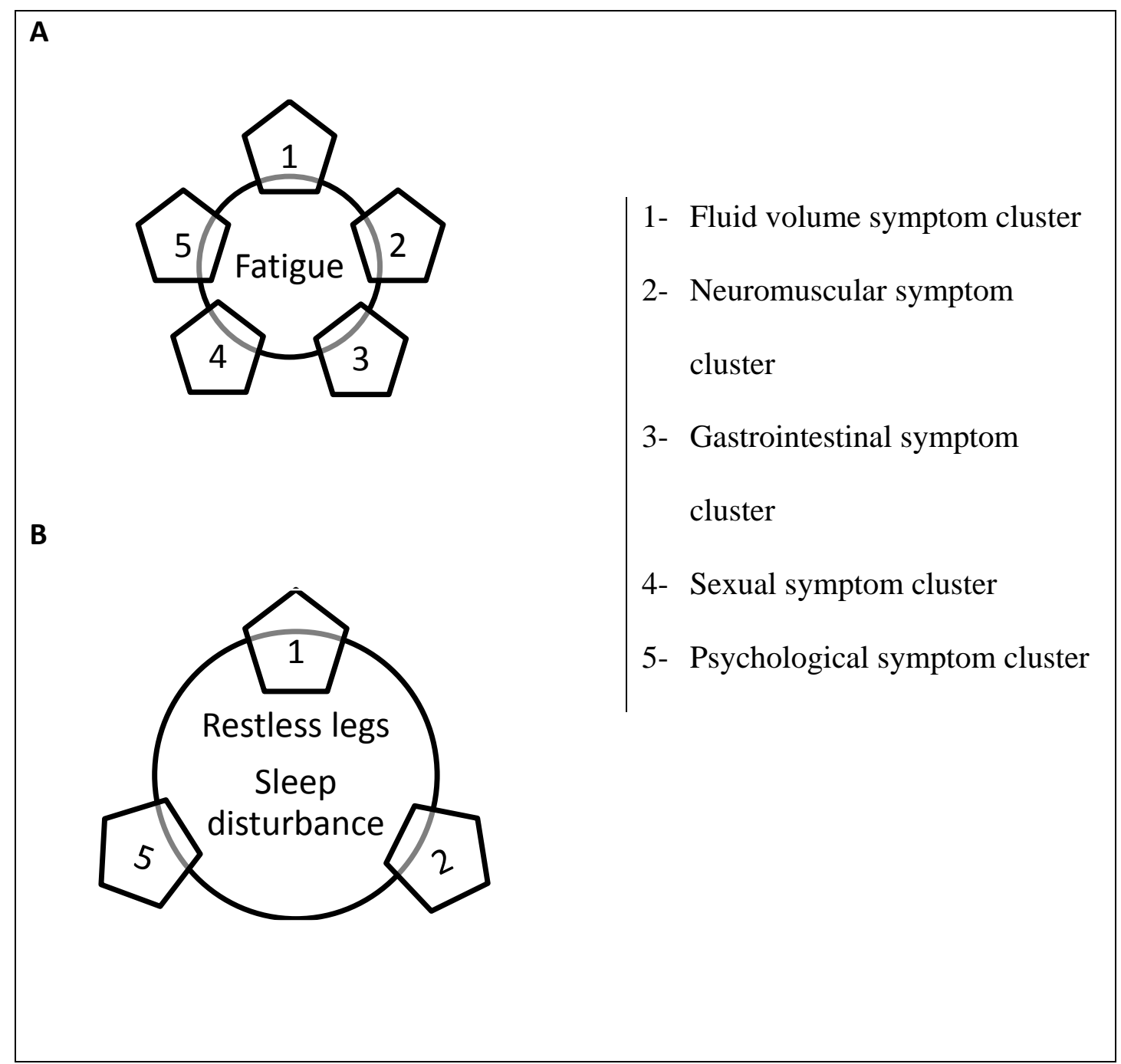

Figure 1. Inter-connecting Symptoms Relationships with CKD Symptom Clusters 


\section{DISCUSSION}

The findings provide evidence that symptoms in CKD appear in clusters rather than in isolation. This study builds on previous research in important ways: we assessed a wider range of physical and psychological symptoms that are experienced by people with advanced stages of CKD; identified and compared the symptom clusters across different symptom dimensions to determine core symptoms that could be clinically significance in identification of symptom clusters; interpreted both the pattern and structure coefficients; and, identified possible interconnecting symptoms through retaining symptoms associated with multiple clusters. Our findings suggest the existence of several coherent and stable symptom clusters across each symptom dimension.

In the fluid volume symptom cluster, there were five core symptoms that reflected the issues related to fluid volume imbalance among CKD patients. Fluid retention is a common clinical feature in advanced stages of CKD due to the elevated extracellular fluid volume as a result of disturbances in the regulation of sodium and water balance and reduced residual kidney function (Hung, Lai, Kuo, \& Tarng, 2015). Excessive accumulation of fluid also contributes to volume overload which may lead to the development of pulmonary oedema (Hryciw \& Bonner, 2015), hypoxia (leading to difficulty concentrating), exacerbation of hypertension and over long periods, alterations in cardiac output (Weiner \& Sarnak, 2010). There is evidence to suggest that fluid volume overload is a predictor of CKD progression (Tsai et al., 2014) and it is also associated with poor survival and increased risk of cardiovascular mortality among dialysis patients (Kalantar-Zadeh et al., 2009). Thus, the fluid volume symptom cluster reflects the impact of fluid imbalance on multiple systems in the body. This underscores the clinical significance of this cluster and suggests planning an effective fluid management strategy (e.g. development of educational materials that are appropriate for different levels of literacy) to target a group of symptoms in this cluster. 
Most patients with CKD experience gastrointestinal symptoms, especially nausea, vomiting and anorexia that associated with uraemia and electrolytes disturbances (Almeras \& Argilés, 2009; Chonchol \& Chan, 2010). This study provides evidence that symptoms related to gastrointestinal function occur in a unique cluster. Previous studies in CKD found symptoms related to the gastrointestinal system were grouped with a different variety of symptoms. For example, $\mathrm{Yu}$ et al. (2012) found that gastrointestinal symptoms in haemodialysis patients were combined with symptoms related to pulmonary function (chest tightness, chest pain) in one cluster. Other studies found that gastrointestinal symptoms grouped with other symptoms such as chest pain, dizziness/faintness, feeling squeezed out and shortness of breath in one cluster (Amro et al., 2014; Thong et al., 2009). Variations in the results may be because these previous studies assessed only one dimension of a few symptoms. Multidimensional assessment of a wide range of symptoms provides better judgment about clustering of symptoms.

We found that muscle soreness and numbness or tingling in feet have occurred concurrently, which reflect impairments in the peripheral nervous system due to impaired renal function (and potentially other comorbid conditions including diabetes, hypertension and peripheral vascular disease typical in late stages of CKD). This cluster has been recognised previously among people receiving dialysis (Amro et al., 2014; Thong et al., 2009). Yu et al. (2012) found a cluster that involved four symptoms (joint pain, arrhythmia, restless leg and numbness) and categorised it as the 'electrolyte imbalance symptom cluster' due to the positive correlation found between this cluster and elevated potassium levels. Reduced renal function results in severe imbalances in electrolyte and mineral metabolism that can affect central nervous system and peripheral neuromuscular transmission (Chonchol \& Chan, 2010). However, we believe that a multifactorial aetiology could contribute to the existence of this cluster. For example, increased ultrafiltration and decreased sodium 
concentration in the dialysate are associated with muscle soreness and cramps (Raymond \& Wazny, 2011).

Another interesting cluster found in this study concerned sexual symptoms. Symptoms related to decreased sexual function are common and are among the most severe and frequent symptoms among patients with CKD (Almutary et al., 2016). Sexual function diminishes with progression of CKD, especially after initiation of dialysis treatment (Nassir, 2009). However, this cluster has not been discovered in previous studies and this probably reflects the fact that sexual symptoms are not routinely measured and neither assessed clinically. Factors that may contribute to genesis of impotence include autonomic neuropathy, increased age, diabetes, hypertension and medication-related side effects (Arieff, 2004). Several studies also suggest strong correlations between sexual function and psychological stressors such as depression (Navaneethan et al., 2010; Yu-Sen et al., 2007). This correlation may raise an interesting research question about relationships between this cluster and psychological symptom clusters. Does providing good support and management for psychological symptoms in CKD patients directly or indirectly improve the severity of the sexual dysfunction symptom cluster? Further investigation is needed in this area.

We found a distinctive cluster related to the psychological symptoms. Psychological status is well known to influence patients' symptom experience, yet except for one study (Lee \& Jeon, 2015), the previous studies in CKD symptom clusters focused only on the physical symptoms. Living with CKD imposes many stressors that can raise the psychological and emotional burden, including uncertainty about the future, change in lifestyle, loss of control, diet and fluid restrictions, extensive drug regimen and the burden related to changes in the social domain of everyday life (Kimmel, Cohen, \& Peterson, 2008; Zalai, Szeifert, \& Novak, 2012). Increasing evidence suggests that a patient's emotional profile, such as anxiety, can largely be influenced by their perception of illness and their coping strategies (Nowak \& 
Laudański, 2014). There is some evidence about the efficacy of psychotherapy and cognitive-behavioural therapy on the management of depression and anxiety among CKD patients (Hedayati, Yalamanchili, \& Finkelstein, 2012). Effective intervention strategies at all stages of the CKD trajectory to target the psychological symptom cluster as a means of improving coping strategies, self-efficacy and self-management behaviours may prove useful.

Compared with previous studies, we identified several symptoms that demonstrated high loadings across multiple clusters. Fatigue was strongly connected with all five symptom clusters, whereas sleep disturbance and restless leg were interconnecting symptoms in three clusters. These findings suggest a more complex pattern of symptom clusters than has been found elsewhere, which may reflect the high symptom burden of CKD compared with other chronic conditions. We have conceptualised a reciprocal relationship between interconnecting symptoms such as fatigue and CKD symptom clusters. Future research using more sophisticated analytics such as hierarchical modelling is needed to understand these relationships further. Fatigue is consistently reported to be a highly prevalent and burdensome symptom among CKD patients (Almutary et al., 2013; Murtagh et al., 2007). It is a complex and highly subjective symptom and may relate to the high symptom burden of CKD. Levels of fatigue reported among people with CKD are comparable to those with terminal stages of cancer (Saini et al., 2006). Evidence based management of fatigue in different chronic illnesses has been identified, such as cancer-related fatigue (Chan, Yates, \& McCarthy, 2011; Mock, 2004). While there are recent discussions on the management of fatigue in CKD (Artom, Moss-Morris, Caskey, \& Chilcot, 2014; Bossola, Vulpio, \& Tazza, 2011; Horigan, Schneider, Docherty, \& Barroso, 2013), CKD-related fatigue management is still a challenging issue for patients and renal clinicians and is an under researched area. This study strengthens the evidence for a bidirectional and potentially causative influence of fatigue and symptom clusters on one another. Based on these findings, we argue that 
comprehensive CKD symptom management programs at the cluster level are needed to reduce pervasive symptoms such as fatigue.

Identification of symptom clusters has significant implications for the development of appropriate intervention strategies which will help in simplifying treatment methods, reducing potential side effects associated with treatments, improving patients' outcomes, prioritising management actions and improving cost effectiveness (Williams, 2007). There is increasing evidence in cancer research to manage a cluster of symptoms (Chan, Richardson, \& Richardson, 2011; Kwekkeboom et al., 2012; Yorke et al., 2015). For example, implementing a psycho-educational intervention in cancer patients has decreased the severity of fatigue, anxiety and breathlessness at the same time (Chan et al., 2011). We argue adopting a symptom cluster approach would also prove effective in controlling CKD symptoms. Moreover, careful assessment and management of CKD symptom clusters may indirectly contribute to the management of fatigue, sleep disturbance and restless leg in CKD patients.

\section{STRENGTHS AND LIMITATIONS}

Our findings support the existence of clinically meaningful symptom clusters in people with advanced CKD and provide an important evidential platform for future symptom research; nevertheless there were several limitations. First, the cross-sectional design was not able to account for the dynamic nature of symptoms along the long disease trajectory often experienced by people with CKD. A longitudinal design to assess the possible changes in symptom clusters over time is warranted. Second, the sample was drawn from one country. While it is likely that similar symptom clusters will be found across cultures (Moser et al., 2014), validation of the findings with diverse samples would strengthen the generalizability of results. Third, the focus of this study was at the symptom rather than individual level. Identification of different subgroups of people with CKD according to their experiences of 
specific symptom clusters is an important area for future research (Miaskowski, Aouizerat, Dodd, \& Cooper, 2007) that could assist clinicians to target patients at risk for greater symptom burden and poorer outcomes. Finally, testing potential causal mechanisms by modelling the associations between symptom clusters, potential influencing factors (e.g. age, co-morbid conditions) and consequences (e.g. functional capacity, quality of life) would also validate these findings and extend our understanding of the CKD symptom experience.

\section{CONCLUSION}

Adopting a symptom cluster approach has the potential to significantly advance symptom assessment and management in CKD. Nurses should be aware of the five possible symptom clusters that are associated with advanced stages of CKD. Routine clinical assessment and management strategies targeted at the cluster level should have synergistic effects in reducing symptoms. Fatigue is a highly prevalent and pervasive symptom for those with CKD that is interconnected with global symptom burden, suggesting better management of symptom clusters may also reduce fatigue. Further exploration of the associations between symptom clusters, potential influencing factors and consequences will contribute to better insights into underlying aetiology which in term may improve treatment. 


\section{References}

Almeras, C., \& Argilés, À. (2009). The general picture of uremia. Seminars in Dialysis, 22(4), 329-333.

Almutary, H., Bonner, A., \& Douglas, C. (2013). Symptom burden in chronic kidney disease: a review of recent literature. Journal of Renal Care, 39(3), 140-150.

Almutary, H., Bonner, A., \& Douglas, C. (2015). Arabic translation, adaptation and modification of the dialysis symptom index for chronic kidney disease stages four and five. BMC Nephrology 16(1), 1-8.

Almutary, H., Bonner, A., \& Douglas, C. (2016). Which people with chronic kidney disease have the greatest symptom burden? a comparative study of advanced CKD stage and dialysis modality. Journal of Renal Care, 42(2), 73-82.

Amro, A., Waldum, B., Dammen, T., Miaskowski, C., \& Os, I. (2014). Symptom clusters in patients on dialysis and their association with quality-of-life outcomes. Journal of Renal Care, 40(1), 23-33.

Amro, A., Waldum, B., Lippe, N. v. d., Brekke, F. B., Dammen, T., Miaskowski, C., \& Os, I. (2015). Symptom clusters predict mortality among dialysis patients in Norway: A prospective observational cohort study. Journal of Pain and Symptom Management, 49(1), 27-35.

Arieff, A. (2004). Neurologic complications of renal insufficiency. In B. Brenner, M. (Ed.), Brenner and Rector's The Kidney ( $7^{\text {th }}$ ed.). Philadelphia: W.B. Saunders.

Artom, M., Moss-Morris, R., Caskey, F., \& Chilcot, J. (2014). Fatigue in advanced kidney disease. Kidney International, 86(3), 497-505. 
Bossola, M., Vulpio, C., \& Tazza, L. (2011). Fatigue in chronic dialysis patients. Seminars in Dialysis, 24(5), 550-555.

Chan, C. W. H., Richardson, A., \& Richardson, J. (2011). Managing Symptoms in Patients with Advanced Lung Cancer During Radiotherapy: Results of a Psychoeducational Randomized Controlled Trial. Journal of Pain \& Symptom Management, 41(2), 347357.

Chan, R. J., Yates, P., \& McCarthy, A. L. (2011). The aetiology, impact and management of cancer-related fatigue in patients with advanced cancer. Australian Journal of Cancer Nursing, 12(2), 4-11.

Chonchol, M., \& Chan, L. (2010). Chronic kidney disease: manifestations and pathogenesis In R. W. Schrier (Ed.), Renal and Electrolyte Disorders ( $7^{\text {th }}$ ed.). Philadelphia , USA: Wolters Kluwer Health.

Costello, A. B., \& Osborne, J. W. (2005). Best practices in exploratory factor analysis: four recommendations for getting the most from your analysis. Practical Assessment Research \& Evaluation, 10(7), 1-9.

Davies, S. J., Phillips, L., Naish, P. F., \& Russell, G. I. (2002). Quantifying comorbidity in peritoneal dialysis patients and its relationship to other predictors of survival. Nephrology, Dialysis, Transplantation: Official Publication Of The European Dialysis And Transplant Association - European Renal Association, 17(6), 10851092.

Dodd, M. J., Miaskowski, C., \& Lee, K. A. (2004). Occurrence of Symptom Clusters. Journal of the National Cancer Institute. Monographs, 2004(32), 76-76. 
Hair, J., black, W., Babin, B., \& Anderson, R. (2014). Multivariate Data Analysis (7 ${ }^{\text {th }}$ ed.). Harlow: Pearson Education Limited.

Hedayati, S. S., Yalamanchili, V., \& Finkelstein, F. O. (2012). A practical approach to the treatment of depression in patients with chronic kidney disease and end-stage renal disease. Kidney International, 81(3), 247-255.

Herr, J. K., Salyer, J., Flattery, M., Goodloe, L., Lyon, D. E., Kabban, C. S., \& Clement, D. G. (2015). Heart failure symptom clusters and functional status - a cross-sectional study. Journal of Advanced Nursing, 71(6), 1274-1287.

Horigan, A. E., Schneider, S. M., Docherty, S., \& Barroso, J. (2013). The experience and self-management of fatigue in patients on hemodialysis. Nephrology Nursing Journal, 40(2), 113-122.

Hryciw, D., \& Bonner, A. (2015). Alterations of renal and urinary tract function across the life span. In J. Craft, C. Gordon, S. Huether, K. McCance, V. Brashers \& N. Rote (Eds.), Understanding pathophysiology (2 $2^{\text {nd }}$ ed.). Chatswood, N.S.W: Mosby/ Elsevier Australia.

Hung, S.-C., Lai, Y.-S., Kuo, K.-L., \& Tarng, D.-C. (2015). Volume overload and adverse outcomes in chronic kidney disease: clinical observational and animal studies. Journal Of The American Heart Association, 4(5), e001918.

Jablonski, A. (2007). The multidimensional characteristics of symptoms reported by patients on hemodialysis. Nephrology Nursing Journal, 34(1), 29-38.

Johnson, D. W., Jones, G. R. D., Mathew, T. H., Ludlow, M. J., Doogue, M. P., Jose, M. D., . . . Usherwood, T. (2012). Chronic kidney disease and automatic reporting of 
estimated glomerular filtration rate: new developments and revised recommendations. The Medical Journal of Australia, 197(4), 224-225.

Kalantar-Zadeh, K., Regidor, D. L., Kovesdy, C. P., Van Wyck, D., Bunnapradist, S., Horwich, T. B., \& Fonarow, G. C. (2009). Fluid retention is associated with cardiovascular mortality in patients undergoing long-term hemodialysis. Circulation, 119(5), 671-679.

Kim, H.-J., McGuire, D., Tulman, L., \& Barsevick, A. (2005). Symptom clusters: concept analysis and clinical implications for cancer nursing. Cancer Nursing, 28(4), 270-284.

Kimmel, P. L., Cohen, S. D., \& Peterson, R. A. (2008). Depression in patients with chronic renal disease: where are we going? Journal of Renal Nutrition, 18(1), 99-103.

Kwekkeboom, K. L., Abbott-Anderson, K., Cherwin, C., Roiland, R., Serlin, R. C., \& Ward, S. E. (2012). Pilot randomized controlled trial of a patient-controlled cognitivebehavioral intervention for the pain, fatigue, and sleep disturbance symptom cluster in cancer. Journal of Pain and Symptom Management, 44(6), 810-822.

Lee, S. J., \& Jeon, J. (2015). Relationship between symptom clusters and quality of life in patients at stages 2 to 4 chronic kidney disease in Korea. Applied Nursing Research: ANR, 28(4), e13-e19.

Lenz, E. R., Pugh, L. C., Milligan, R. A., Gift, A., \& Suppe, F. (1997). The middle-range theory of unpleasant symptoms: an update. Advances in Nursing Science, 19(3), 1427.

Miaskowski, C., Aouizerat, B. E., Dodd, M., \& Cooper, B. (2007). Conceptual issues in symptom clusters research and their implications for quality-of-life assessment in patients with cancer. JNCI: Journal of the National Cancer Institute(37), 39-46. 
Mock, V. (2004). Evidence-based treatment for cancer-related fatigue. JNCI: Journal of the National Cancer Institute, 96(1), 112-118.

Moser, D. K., Kyoung Suk, L., Jia-Rong, W., Gia, M.-M., Tiny, J., Tsuey-Yuan, H., . . . Riegel, B. (2014). Identification of symptom clusters among patients with heart failure: An international observational study. International Journal of Nursing Studies, 51(10), 1366-1372.

Murtagh, F., Addington-Hall, J., \& Higginson, I. J. (2007). The prevalence of symptoms in end-stage renal disease: a systematic review. Advances in Chronic Kidney Disease, 14(1), 82-99.

Nassir, A. (2009). Sexual function in male patients undergoing treatment for renal failure: a prospective view. The Journal of Sexual Medicine, 6(12), 3407-3414.

Navaneethan, S. D., Vecchio, M., Johnson, D. W., Saglimbene, V., Graziano, G., Pellegrini, F., . . Bonifati, C. (2010). Prevalence and correlates of self-reported sexual dysfunction in CKD: a meta-analysis of observational studies. American Journal of Kidney Diseases, 56(4), 670-685.

Nowak, Z., \& Laudański, K. (2014). The perception of the illness with subsequent outcome measure in more favorable in continuos peritoneal dialysis vs hemodialysis in the framework of appraisal model of stress. International Journal of Medical Sciences, 11(3), 291-297.

Raymond, C. B., \& Wazny, L. D. (2011). Treatment of leg cramps in patients with chronic kidney disease receiving hemodialysis. CANNT Journal, 21(3), 19-23. 
Saini, T., Murtagh, F. E. M., Dupont, P. J., McKinnon, P. M., Hatfield, P., \& Saunders, Y. (2006). Comparative pilot study of symptoms and quality of life in cancer patients and patients with end stage renal disease. Palliative Medicine, 20(6), 631-636.

Skerman, H., Yates, P., \& Battistutta, D. (2009). Multivariate methods to identify cancerrelated symptom clusters. Research in Nursing \& Health, 32(3), 345-360.

Skerman, H., Yates, P., \& Battistutta, D. (2012). Identification of cancer-related symptom clusters: an empirical comparison of exploratory factor analysis methods. Journal of Pain And Symptom Management, 44(1), 10-22.

Tabachnick, B. G., \& Fidell, L. S. (2014). Using multivariate statistics. (6 ${ }^{\text {th }}$ ed.). Harlow: Pearson Education Limited.

Thong, M. S. Y., van Dijk, S., Noordzij, M., Boeschoten, E. W., Krediet, R. T., Dekker, F. W., \& Kaptein, A. A. (2009). Symptom clusters in incident dialysis patients: associations with clinical variables and quality of life. Nephrology, Dialysis, Transplantation: Official Publication of the European Dialysis and Transplant Association - European Renal Association, 24(1), 225-230.

Tsai, Y.-C., Tsai, J.-C., Chen, S.-C., Chiu, Y.-W., Hwang, S.-J., Hung, C.-C., . . Chen, H.-C. (2014). Association of Fluid Overload With Kidney Disease Progression in Advanced CKD: A Prospective Cohort Study. American Journal of Kidney Diseases, 63(1), 6875.

Weiner, D., \& Sarnak, M. (2010). Cardiovascular disease in patients with chronic kidney disease. In J. Himmelfarb \& M. Sayegh (Eds.), Chronic kidney disease, dialysis, and transplantation ( $3^{\text {th }}$ ed.). Philadelphia, PA: Saunders, an imprint of Elsevier Inc. 
Weisbord, S., Fried, L. F., Arnold, R. M., Rotondi, A. J., Fine, M. J., Levenson, D. J., \& Switzer, G. E. (2004). Development of a symptom assessment instrument for chronic hemodialysis patients: The Dialysis Symptom Index. Journal of Pain \& Symptom Management, 27(3), 226-240.

Williams, L. A. (2007). Clinical management of symptom clusters. Seminars in Oncology Nursing, 23(2), 113-120.

Yorke, J., Lloyd-Williams, M., Smith, J., Blackhall, F., Harle, A., Warden, J., . . . Molassiotis, A. (2015). Management of the respiratory distress symptom cluster in lung cancer: a randomised controlled feasibility trial. Supportive Care in Cancer: Official Journal of the Multinational Association of Supportive Care in Cancer, 23(11), 3373-3384.

Yu-Sen, P., Chih-Kang, C., Kung-Yu, H., Shou-Shang, C., Chia-Sheng, L., Chwei-Shiun, Y., .. . Tun-Jun, T. (2007). The association of higher depressive symptoms and sexual dysfunction in male haemodialysis patients. Nephrology Dialysis Transplantation, 22(3), 857-861.

Yu, I. C., Huang, J.-Y., \& Tsai, Y.-F. (2012). Symptom cluster among hemodialysis patients in Taiwan. Applied Nursing Research, 25(3), 190-196.

Zalai, D., Szeifert, L., \& Novak, M. (2012). Psychological distress and depression in patients with chronic kidney disease. Seminars in Dialysis, 25(4), 428-438. 